ESAIM: PROCEEDINGS, April 2009, Vol. 26, p. 207-216

H. Ammari, Editor

\title{
DETECTION OF INTERNAL CORROSION
}

\author{
H. Ammari ${ }^{1}, \mathrm{H} \cdot \mathrm{KANG}^{2}$ AND E. KIM ${ }^{3}$
}

Abstract. The purpose of this paper is to review recently developed algorithms for corrosion detection and to compare between them.

\section{INTRODUCTION}

Corrosion detection is an important problem in the field of nondestructive evaluation (NDE) for an obvious practical reason, and new techniques are constantly being sought for the detection of hidden corrosion. In relation to the NDE of the corrosion there have been several mathematical works: See, for example, Kaup and Santosa [8], Kaup et al. [9], Vogelius and $\mathrm{Xu}$ [11], Inglese [7], Luong and Santosa [10], Banks et al. [4] and references therein.

In general corrosion is defect in various phenomena such as electrical defect or defect for the wave propagation or the guided wave. Therefore the methods of detecting corrosion can vary depending on the related physical phenomena. Quite recently various algorithms for corrosion detection are proposed and tested numerically in three different models: the electrostatic model [3], the ultrasound model [2], and the guided wave model [1]. The purpose of this paper is to review the results of these works and compare them.

\section{Mathematical MOdels}

The electrostatic model of corrosion of this paper is taken from [11], which we review briefly here. Let $\Omega$ be a bounded domain in $\mathbb{R}^{d}, d=2,3$, and suppose that a corrosion occurs on a part $\Gamma$ of the boundary $\partial \Omega$ of $\Omega$. Vogelius and Xu derived in [11] that the electrostatic potential $u$ in the presence of corrosion is a solution of the following nonlinear

1 Institut Langevin, Laboratoire Ondes et Acoustique, CNRS UMR 7587, ESPCI, 10 rue Vauquelin, 75231 Paris Cedex 05, France; email: habib.ammari@polytechnique.fr

2 Department of Mathematics, Inha University, Incheon 402-751, Korea; email: hbkang@inha.ac.kr

${ }^{3}$ Ewha Women's University, Seoul 120-750, Korea; email: kim123ej@hanmail.net

(c) EDP Sciences, SMAI 2009

Article published by EDP Sciences and available at http://www.edpsciences.org/proc or http://dx.doi.org/10.1051/proc/2009014 
boundary value problem:

$$
\begin{cases}\Delta u=0 & \text { in } \Omega, \\ \frac{\partial u}{\partial \nu}+\gamma\left(e^{\alpha u}-e^{(\alpha-1) u}\right)=0 & \text { on } \Gamma, \\ \frac{\partial u}{\partial \nu}=g & \text { on } \partial \Omega \backslash \Gamma\end{cases}
$$

for some constant $\gamma$ and $\alpha$, provided that a current flux $g$ is applied on $\partial \Omega \backslash \Gamma$. This nonlinear elliptic equation is derived from the so-called Butler-Volmer formula. By linearizing this nonlinear problem around $u=0$, the following linear elliptic equation is derived:

$$
\begin{cases}\Delta u=0 & \text { in } \Omega, \\ \frac{\partial u}{\partial \nu}+\gamma u=0 & \text { on } \Gamma, \\ \frac{\partial u}{\partial \nu}=0 & \text { on } \partial \Omega \backslash \Gamma,\end{cases}
$$

where $\nu$ is the outward unit normal to $\Omega$ on $\partial \Omega$. Note that the $\gamma$ in $(2.2)$ is $(2 \alpha-1)$ times $\gamma$ in (2.1). What is important in (2.2) is that the corrosive part of the boundary is characterized by a Robin type boundary condition.

We will be considering a cross section of three dimensional pipeline inside which there are corrosive parts. Thus the specimen $\Omega$ to be inspected in this paper is a two dimensional annulus. To be precise, $\Omega=U \backslash \bar{D}$, where $U$ is a simply connected bounded $\mathcal{C}^{2}$ domain in $\mathbb{R}^{2}$ and $D$ is a simply connected $\mathcal{C}^{2}$ domain compactly contained in $U$. We define $\Gamma_{e}=\partial U$ and $\Gamma_{i}=\partial D$ so that $\partial \Omega=\Gamma_{i} \cup \Gamma_{e}$, where $\Gamma_{e}$ and $\Gamma_{i}$ are the external and internal boundaries of $\Omega$, respectively. Suppose that the inaccessible surface $\Gamma_{i}$ contains some corrosive parts $I_{s}, s=1, \ldots, m$. We assume that the parts $I_{s}$ are well-separated and the reciprocal of the surface impedance (the corrosion coefficient) of each $I_{s}, s=1, \ldots, m$, is $\gamma_{s} \geq 0$, not identically zero. We also assume that each $\gamma_{s} \in \mathcal{C}^{1}\left(I_{s}\right)$. Let

$$
\gamma(x)=\sum_{s=1}^{m} \gamma_{s} \chi_{s}(x), \quad x \in \Gamma_{i}
$$

where $\chi_{s}$ denotes the characteristic function on $I_{s}$. A significant assumption on the corrosion is smallness: We assume that the one-dimensional Hausdorff measures of $I_{s}$ are small:

$$
\left|I_{s}\right|=O(\epsilon), \quad s=1, \ldots, m,
$$

where $\epsilon$ is a small parameter representing the common order of magnitude of $I_{s}$. Here and throughout this paper $|\cdot|$ denotes the one-dimensional Hausdorff measure. It is worth emphasizing that the smallness assumption is on $I_{s}$, not on the corrosion coefficient $\gamma_{s}$.

According to $(2.2)$, the voltage potential $u_{\epsilon}$ generated by a voltage $f$ applied on $\Gamma_{e}$ satisfies

$$
\begin{cases}\Delta u_{\epsilon}=0 & \text { in } \Omega \\ -\frac{\partial u_{\epsilon}}{\partial \nu}+\gamma u_{\epsilon}=0 & \text { on } \Gamma_{i} \\ u_{\epsilon}=f & \text { on } \Gamma_{e}\end{cases}
$$


where $\nu$ is the outward unit normal to $\Omega$ on $\Gamma_{e}$ and inward on $\Gamma_{i}$.

Another justification of the mathematical model can be found in the work of Buttazzo and Kohn. The corrosion can be regarded as a thin coating and it is proved in [5] that as the thickness of the coating approaches to zero the boundary condition tends to the Robin boundary condition in (2.5). This justification of the model may be applied to other kinds of corrosion such as the one for the ultrasound wave and the one for the eigen-modes.

The ultrasound wave $u_{\epsilon}$ generated by a source at $y \in \Gamma_{e}$ in the presence of the corrosion satisfies

$$
\begin{cases}\left(\Delta+\omega^{2}\right) u_{\epsilon}=0 & \text { in } \Omega \\ -\frac{\partial u_{\epsilon}}{\partial \nu}+\gamma u_{\epsilon}=0 & \text { on } \Gamma_{i} \\ u_{\epsilon}=\Phi_{\omega}(\cdot-y) & \text { on } \Gamma_{e}\end{cases}
$$

where $\omega$ is the frequency of the wave and $\Phi_{\omega}$ is the fundamental solution to the Helmholtz equation, i.e.,

$$
\Phi_{\omega}(x)=-\frac{i}{4} H_{0}^{(1)}(\omega|x|), \quad x \neq 0 .
$$

Here $H_{0}^{(1)}$ is the Hankel function of the first kind of order 0 [6].

On the other hand, the eigenvalue problem in the presence of corrosion consists of finding $\omega_{\epsilon}>0$ such that there exists a nontrivial solution $v_{\epsilon}$ to

$$
\begin{cases}\left(\Delta+\omega_{\epsilon}^{2}\right) v_{\epsilon}=0 & \text { in } \Omega \\ -\frac{\partial v_{\epsilon}}{\partial \nu}+\gamma \chi(I) v_{\epsilon}=0 & \text { on } \Gamma_{i} \\ v_{\epsilon}=0 & \text { on } \Gamma_{e} \\ \int_{\Omega} v_{\epsilon}^{2}=1, & \end{cases}
$$

assuming that there is a single corrosive part $I$.

The inverse problem considered in the papers [1-3] is to detect the corrosive parts on the inaccessible boundary $\Gamma_{i}$ by means of the measurements on the accessible boundary $\Gamma_{e}$. In particular, the aim is to detect the location and the size of the corrosive parts, and the corrosion coefficient $\gamma_{s}$. For the problem (2.5) and (2.6) the measurements are $\frac{\partial u_{\epsilon}}{\partial \nu}$ on $\Gamma_{e}$, while for the problem $(2.8)$ the measurement is the pair of modal parameters $\left(\omega_{\epsilon},\left.\frac{\partial v_{\epsilon}}{\partial \nu}\right|_{\Gamma_{e}}\right)$. In above mentioned works, various algorithms to detect corrosive parts are proposed and tested numerically. Those algorithms are based on asymptotic expansions of the solutions of the related equations which we review in the next section.

\section{Asymptotic EXPANsions}

We now review the asymptotic expansions of the measurements as the smallness parameter $\epsilon$ tends to zero. 
Electrostatic measurements: Let $G(x, z)$ be the Green function for the problem (2.5) with $\gamma=0$, i.e., for each $z \in \Omega, G(x, z)$ is the solution to

$$
\begin{cases}\Delta_{x} G(x, z)=-\delta_{z} & \text { in } \Omega, \\ \frac{\partial}{\partial \nu_{x}} G(x, z)=0, & x \in \Gamma_{i}, \\ G(x, z)=0, & x \in \Gamma_{e} .\end{cases}
$$

Let $u_{\epsilon}$ be the solution to (2.5) and $u_{0}$ the one when $\gamma=0$, i.e., in the absence of the corrosion. Then the following asymptotic expansion holds uniformly for $x \in \Gamma_{e}[3]$ :

$$
\frac{\partial u_{\epsilon}}{\partial \nu}(x)=\frac{\partial u_{0}}{\partial \nu}(x)-\sum_{s=1}^{m}\langle\gamma\rangle_{s} u_{0}\left(z_{s}\right) \frac{\partial}{\partial \nu_{x}} G\left(x, z_{s}\right)+O\left(\epsilon^{1+\alpha}\right)
$$

for some $\alpha>0$, where

$$
\langle\gamma\rangle_{s}:=\int_{I_{s}} \gamma d s
$$

and $z_{s}$ denotes the location of $I_{s}, s=1, \ldots, m$.

Ultrasound measurements: Let $G_{\omega}$ be the Green function for the problem (2.6) with $\gamma=0$, i.e., for each $z \in \Omega, G_{\omega}(x, z)$ is the solution to

$$
\begin{cases}\left(\Delta_{x}+\omega^{2}\right) G_{\omega}(x, z)=-\delta_{z} & \text { in } \Omega, \\ \frac{\partial G_{\omega}}{\partial \nu_{x}}(x, z)=0, & x \in \Gamma_{i}, \\ G_{\omega}(x, z)=0, & x \in \Gamma_{e} .\end{cases}
$$

Let $u_{\epsilon}$ be the solution to (2.6) and $u_{0}$ the one when $\gamma=0$, i.e., in the absence of the corrosion. Suppose that the wavelength $\lambda=2 \pi / \omega=\epsilon^{1-\beta}(>>\epsilon)$ for some positive constant $\beta$, then the following asymptotic formula holds uniformly on $\Gamma_{e}$ for some positive constant $\alpha[3]$ :

$$
\frac{\partial u_{\epsilon}}{\partial \nu}(x)=\frac{\partial u_{0}}{\partial \nu}(x)-\sum_{s=1}^{m}\langle\gamma\rangle_{s} u_{0}\left(z_{s}\right) \frac{\partial G_{\omega}}{\partial \nu}\left(x, z_{s}\right)+O\left(\epsilon^{1+\alpha}\right), \quad x \in \Gamma_{e} .
$$

Note that (3.2) is at least formally the limiting case $(\omega=0)$ of $(3.4)$.

Modal parameter measurements: Suppose there is a single corrosive part $I$ and the corrosion coefficient $\gamma$ is constant. Let $\omega_{\epsilon}$ and $v_{\epsilon}$ be an eigenvalue of (2.8) and the corresponding (normalized) eigenfunction, respectively, and let $\omega_{0}$ and $v_{0}$ be those in the absence of the corrosion. Then the following asymptotic formula were derived in [1]:

$$
\omega_{\epsilon}=\omega_{0}+\frac{\gamma}{2 \omega_{0}} \int_{I} v_{0}^{2}+O\left(\epsilon^{2}\right)
$$

as $\epsilon \rightarrow 0$. Furthermore,

where $O(\epsilon)$ is in $H^{3 / 2}(\Omega)$-norm.

$$
v_{\epsilon}=v_{0}+O(\epsilon)
$$


Rigorous derivations of asymptotic expansions can be found in [1-3]. The expansions (3.5) and (3.6) can be seen easily by expanding $\omega_{\epsilon}$ and $v_{\epsilon}$ formally as

$$
\begin{gathered}
\omega_{\epsilon}=\omega_{0}+\epsilon \omega_{1}+\epsilon^{2} \omega_{2}+\ldots, \\
v_{\epsilon}=v_{0}+\epsilon v_{1}+\epsilon^{2} v_{2}+\ldots
\end{gathered}
$$

\section{Corrosion Detection Algorithms}

We now describe three algorithms for detection of internal corrosive parts. They are MUSIC-type algorithms and the vibration test algorithm, and based on the asymptotic expansions explained in the previous section. Since the MUSIC-type algorithms for (2.5) [3] and (2.6) [2] are almost identical, we will only describe the one using the ultrasound measurements.

MUSIC type algorithm. Let, for $z \in \Gamma_{i}$ and $x \in \Gamma_{e}$,

$$
h_{z}(x):=\int_{\Gamma_{e}} \frac{\partial G_{\omega}}{\partial \nu}(y, z) \Phi_{\omega}(y-x) d \sigma(y) .
$$

Then the following is the MUSIC characterization of the location of the corrosive parts, a proof of which can be found in [2]:

MUSIC characterization. If there are complex numbers $a_{1}, \ldots, a_{m}$ such that

$$
h_{z}(y)=\sum_{s=1}^{m} a_{s} h_{z_{s}}(y) \quad \text { for all } y \in \Gamma_{e}
$$

then $z \in\left\{z_{1}, \ldots, z_{m}\right\}$.

Let us now discretize the relation (4.1) with sampling points $\left\{y_{1}, \ldots, y_{N}\right\}$ on $\Gamma_{e}$. Let

$$
v_{s}=\left(h_{z_{s}}\left(y_{1}\right), \ldots, h_{z_{s}}\left(y_{N}\right)\right)^{T}, \quad s=1, \ldots, m
$$

where $T$ denotes the transpose. Then the discrete version of (4.1) is

$$
\left(h_{z}\left(y_{1}\right), \ldots, h_{z}\left(y_{N}\right)\right)^{T} \text { is a linear combination of }\left\{v_{1}, \ldots, v_{m}\right\} \text {. }
$$

So the question now is how to characterize those points $z$ such that (4.3) holds. It should be emphasized that the values $h_{z_{s}}\left(y_{j}\right)$ are not known since the location of corrosive parts $z_{s}$ is not known. But we claim that (4.3) can be checked using the boundary measurements and the asymptotic formula (3.4) plays a central role in this, as we see in the following.

Let us first introduce the notion of the Dirichlet-to-Neumann (DtN) map. Let $u$ be the solution to the problem

$$
\begin{cases}\left(\Delta+\omega^{2}\right) u=0 & \text { in } \Omega \\ -\frac{\partial u}{\partial \nu}+\gamma u=0 & \text { on } \Gamma_{i} \\ u=f & \text { on } \Gamma_{e}\end{cases}
$$


and define the DtN map $\Lambda_{\gamma}^{\omega}$ by

$$
\Lambda_{\gamma}^{\omega}(f):=\left.\frac{\partial u}{\partial \nu}\right|_{\Gamma_{e}}
$$

Let $\Lambda_{0}^{\omega}$ be the DtN map when there is no corrosion.

By the definition of the Green function $G_{\omega}$, we have $h_{z}(x)=-u_{0}(z)$ where $u_{0}$ is the solution to

$$
\begin{cases}\left(\Delta+\omega^{2}\right) u_{0}=0 & \text { in } \Omega \\ \frac{\partial u_{0}}{\partial \nu}=0 & \text { on } \Gamma_{i} \\ u_{0}=\Phi_{\omega}(\cdot-x) & \text { on } \Gamma_{e}\end{cases}
$$

Thus it follows from (3.4) that

$$
\left(\Lambda_{\gamma}^{\omega}-\Lambda_{0}^{\omega}\right)\left(\Phi_{\omega}(\cdot-x)\right)(y) \approx \sum_{s=1}^{m}\langle\gamma\rangle_{s} h_{z_{s}}(x) \frac{\partial G_{\omega}}{\partial \nu}\left(y, z_{s}\right), \quad x, y \in \Gamma_{e}
$$

By integrating both sides of the above relation against $\Phi_{\omega}(\cdot-t)$ for $t \in \Gamma_{e}$, we obtain

$$
\int_{\Gamma_{e}}\left(\Lambda_{\gamma}^{\omega}-\Lambda_{0}^{\omega}\right)\left(\Phi_{\omega}(\cdot-x)\right)(y) \Phi_{\omega}(y-t) d \sigma(y) \approx \sum_{s=1}^{m}\langle\gamma\rangle_{s} h_{z_{s}}(x) h_{z_{s}}(t), \quad x, t \in \Gamma_{e} .
$$

In particular, we have

$$
\int_{\Gamma_{e}}\left(\Lambda_{\gamma}^{\omega}-\Lambda_{0}^{\omega}\right)\left(\Phi_{\omega}\left(\cdot-y_{i}\right)\right)(y) \Phi_{\omega}\left(y-y_{j}\right) d \sigma(y) \approx \sum_{s=1}^{m}\langle\gamma\rangle_{s} h_{z_{s}}\left(y_{i}\right) h_{z_{s}}\left(y_{j}\right), \quad i, j=1, \ldots, N
$$

Let $A:=\left(a_{i j}\right)$ where

$$
a_{i j}:=\sum_{s=1}^{m}\langle\gamma\rangle_{s} h_{z_{s}}\left(y_{i}\right) h_{z_{s}}\left(y_{j}\right), \quad i, j=1, \ldots, N
$$

One can easily see that $(4.3)$ is equivalent to

$$
h_{z}:=\left(h_{z}\left(y_{1}\right), \ldots, h_{z}\left(y_{N}\right)\right)^{T} \in \operatorname{Range}(A) .
$$

Let

$$
A^{c}:=\left(\int_{\Gamma_{e}}\left(\Lambda_{\gamma}^{\omega}-\Lambda_{0}^{\omega}\right)\left(\Phi_{\omega}\left(\cdot-y_{i}\right)\right)(y) \Phi_{\omega}\left(y-y_{j}\right) d \sigma(y)\right)_{i, j=1, \ldots, N} .
$$

Note that $A^{c}$ can be computed from the boundary measurements $\left(\Lambda_{\gamma}^{\omega}-\Lambda_{0}^{\omega}\right)\left(\Phi_{\omega}\left(\cdot-y_{i}\right)\right)$. The relation (4.6) shows that $A^{c}$ gives an approximation of $A$. Since $A$ and $A^{c}$ are symmetric matrices, they admit (orthogonal) singular value decompositions (SVD). The number of significant eigenvalues of $A$ is exactly the number of corrosive parts. But we do not have $A$ as our data. Instead we have $A^{c}$ which is only approximation of $A$. Moreover, the SVD of $A^{c}$ does not exhibit a clear drop of the eigenvalues as one can see from Figure 1 . It means that we can not determine the number of corrosive parts from the SVD of $A^{c}$. 

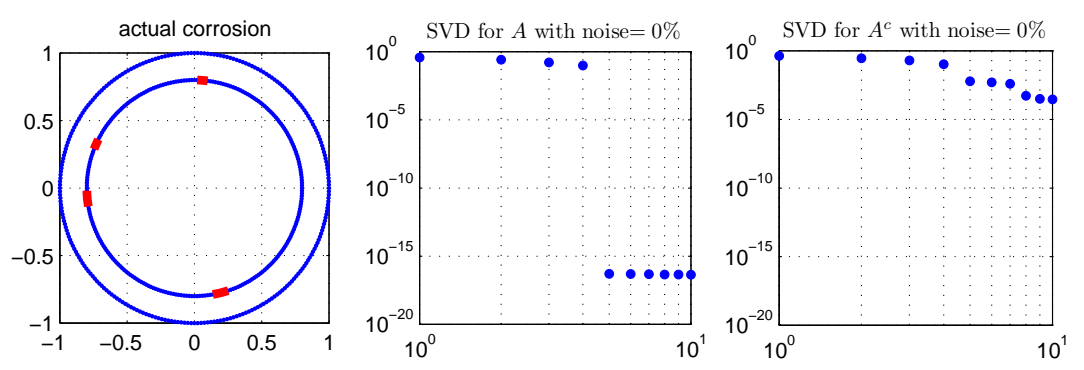

Figure 1. SVD of $A$ and $A^{c}$.

In order to overcome this difficulty we proceed as follows: Let

$$
A^{c}=\sum_{n=1}^{N} \lambda_{n} v_{n} \otimes v_{n}
$$

be the SVD of $A^{c}$ where $\lambda_{1} \geq \lambda_{2} \geq \ldots \geq \lambda_{N}$ are eigenvalues of $A^{c}$ and $v_{n}$ are corresponding eigenvectors. For $k=1,2, \ldots, N$, let $S_{k}$ be the space spanned by $v_{1}, \ldots, v_{k}$, and let $P_{k}$ be the orthogonal projector from $\mathbb{R}^{N}$ onto $S_{k}$. Define $\theta_{k}(z)$ by

$$
\cot \theta_{k}(z)=\frac{\left\|P_{k}^{\epsilon}\left(h_{z}\right)\right\|}{\left\|\left(I-P_{k}^{\epsilon}\right)\left(h_{z}\right)\right\|}, \quad z \in \Gamma_{i}
$$

and compute the minimal value of $\theta_{k}$. We repeat this process for $k=1,2, \ldots$ until the minimal values are stabilized. It turned out that this MUSIC type algorithm detect the number of corrosive parts $m$ and their locations $z_{s}$ pretty well. See $[2,3]$. We will present some computational results in the next section.

Once the locations of the corrosive parts were found, it is straightforward to compute the (averaged) corrosion coefficients $\langle\gamma\rangle_{s}$ using (3.2) and (3.4). We omit this.

We note that the same algorithm works for the electrostatic measurement problem (2.5). It is worth emphasizing that the algorithm is a direct not iterative one in the sense that the computation at each step does not use the results of the previous steps.

Vibration test algorithm. We now review the algorithm for the vibration test which is based on the asymptotic expansion (3.5) and (3.6). This algorithm was proposed and tested numerically in [1].

For $h$ such that $\int_{\Gamma_{e}} h \frac{\partial v_{0}}{\partial \nu}=0$, let $w_{h} \in H^{1}(\Omega)$ be the solution to

$$
\begin{cases}\left(\Delta+\omega_{0}^{2}\right) w_{h}=0 & \text { in } \Omega \\ \frac{\partial w_{h}}{\partial \nu}=0 & \text { on } \Gamma_{i} \\ w_{h}=h & \text { on } \Gamma_{e}\end{cases}
$$


Applying Green's formula, we obtain

$$
\gamma \int_{I} w_{h} v_{\epsilon}=\int_{\Gamma_{i}} w_{h} \frac{\partial v_{\epsilon}}{\partial \nu}=\int_{\Gamma_{e}} h \frac{\partial v_{\epsilon}}{\partial \nu}+\left(\omega_{\epsilon}^{2}-\omega_{0}^{2}\right) \int_{\Omega} v_{\epsilon} w_{h} .
$$

Dividing (4.10) by $\omega_{\epsilon}^{2}-\omega_{0}^{2}$ and using (3.5) we induce

$$
\frac{\int_{I} w_{h} v_{\epsilon}}{\int_{I} v_{0}^{2}}=\frac{1}{\omega_{\epsilon}^{2}-\omega_{0}^{2}} \int_{\Gamma_{e}} h \frac{\partial v_{\epsilon}}{\partial \nu}+\int_{\Omega} v_{\epsilon} w_{h}+O(\epsilon) .
$$

By (3.6), we have

$$
\begin{aligned}
& \int_{I} w_{h} v_{\epsilon}=\int_{I} w_{h} v_{0}+O\left(\epsilon^{2}\right) \\
& \int_{\Omega} v_{\epsilon} w_{h}=\int_{\Omega} v_{0} w_{h}+O(\epsilon) .
\end{aligned}
$$

Therefore, we have

$$
\frac{w_{h}(z)}{v_{0}(z)}-\int_{\Omega} v_{0} w_{h} \approx \frac{1}{2 \omega_{0}\left(\omega_{\epsilon}-\omega_{0}\right)} \int_{\Gamma_{e}} \frac{\partial v_{\epsilon}}{\partial \nu} h .
$$

This is the key observation on which our reconstruction procedure is based. Since we are in possession of $\omega_{\epsilon}-\omega_{0}$ and $\left.\frac{\partial v_{\epsilon}}{\partial \nu}\right|_{\Gamma_{e}}$ by the boundary measurements, the reconstruction algorithm is as follows. Let $h=h_{1}, h_{2}, \ldots, h_{n}$, where $\left\{h_{i}\right\}_{i=1}^{n}$ is a set of $n$ independent functions satisfying $\int_{\Gamma_{e}} h_{i} \frac{\partial v_{0}}{\partial \nu}=0$ for $i=1, \ldots, n$. For any $y \in \Gamma_{i}$ such that $v_{0}(y) \neq 0$ compute $\left(w_{h_{i}} / v_{0}\right)(y)$ and $\int_{\Omega} v_{0} w_{h}$. The point $z$ can be found as the minimum point of

$$
J(z):=\sum_{i=1}^{n}\left|\frac{w_{h_{i}}(z)}{v_{0}(z)}-\int_{\Omega} v_{0} w_{h_{i}}-\frac{1}{2 \omega_{0}\left(\omega_{\epsilon}-\omega_{0}\right)} \int_{\Gamma_{e}} \frac{\partial v_{\epsilon}}{\partial \nu} h_{i}\right| .
$$

\section{NumericAl EXAMPLES}

This section presents numerical results of finding the internal corrosive parts by electrostatic, ultrasound, and vibration tests explained in the previous sections, for the purpose of comparing their performance. In the following, $\Omega \subset \mathbb{R}^{2}$ is assumed to be the annulus centered at $(0,0)$ with radii, $r_{e}$ and $r_{i}$. Let $I_{s}, s=1, \cdots, m$, be the corrosive parts with the corrosive coefficients, $\gamma_{s}$. Let $z_{s}$ and $\langle\gamma\rangle_{s}$ be the center and the integration of corrosive part $I_{s}$, and $z_{s}^{c}$ and $\langle\gamma\rangle_{s}^{c}$ the detected ones.

For computation, we discretize $\Gamma_{e}$ and $\Gamma_{i}$ by

$$
\Gamma_{e}=\left\{r_{e}\left(\cos \theta_{n}, \sin \theta_{n}\right) \mid \theta_{n}=2 \pi(n-1) / N, n=1, \cdots, N\right\}
$$

and

$$
\Gamma_{i}=\left\{r_{i}\left(\cos \theta_{n}, \sin \theta_{n}\right) \mid \theta_{n}=2 \pi(n-1) / N, n=1, \cdots, N\right\}
$$

with $N=256$. In the following example, the outer radius $r_{e}=1$ and the inner one $r_{i}=0.8$. We do process under noise $=0 \%, 1 \%, 5 \%$ and $10 \%$. Here $p \%$ noise means that we add $p \%$ 


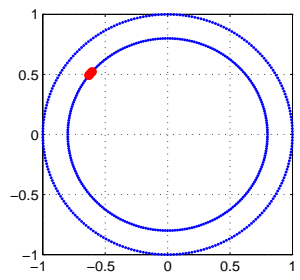

Actual data
\begin{tabular}{|cccc|}
\hline$\gamma_{1}$ & $z_{1}$ & $\langle\gamma\rangle_{1}$ & $\left|I_{1}\right|$ \\
\hline 1 & $(-0.6184,0.5075)$ & 0.0589 & 0.0589 \\
\hline
\end{tabular}

Detected data

\begin{tabular}{|c|cc|cc|cc|}
\hline & \multicolumn{2}{|c|}{ Electrostatic M. } & \multicolumn{2}{c|}{ Ultrasound M. } & \multicolumn{2}{c|}{ Modal Parameter M. } \\
\hline noise(\%) & $\left|z_{1}-z_{1}^{c}\right|$ & $\left|\langle\gamma\rangle_{1}-\langle\gamma\rangle_{1}^{c}\right|$ & $\left|z_{1}-z_{1}^{c}\right|$ & $\left|\langle\gamma\rangle_{1}-\langle\gamma\rangle_{1}^{c}\right|$ & $\left|z_{1}-z_{1}^{c}\right|$ & $\left|\langle\gamma\rangle_{1}-\langle\gamma\rangle_{1}^{c}\right|$ \\
\hline 0 & 0.0000 & $4.8253 \mathrm{e}-4$ & 0.0000 & $3.7965 \mathrm{e}-3$ & 0.0196 & $1.4267 \mathrm{e}-2$ \\
1 & 0.0000 & $4.7000 \mathrm{e}-4$ & 0.0000 & $3.7979 \mathrm{e}-3$ & 0.0393 & $1.3352 \mathrm{e}-2$ \\
5 & 0.0000 & $4.1966 \mathrm{e}-4$ & 0.0000 & $3.7989 \mathrm{e}-3$ & 0.0393 & $9.3961 \mathrm{e}-3$ \\
10 & 0.0000 & $3.5622 \mathrm{e}-4$ & 0.0000 & $3.8013 \mathrm{e}-3$ & 0.0785 & $1.7248 \mathrm{e}-2$ \\
\hline
\end{tabular}

Figure 2. The computational results in the case of a single corrosive part.

random noise of the $L^{2}$-norm of the the measured data $w$ on $\Gamma_{e}$. Thus, when it comes to the electrostatic and ultrasound measurements, the data with $p \%$ noise is the $N \times N$ matrix

$$
\left(\Lambda_{\gamma}-\Lambda_{0}\right)\left(\frac{\partial G}{\partial \nu}\right)+\frac{p}{100} \operatorname{rand}(1) \frac{\left\|\left(\Lambda_{\gamma}-\Lambda_{0}\right)\left(\frac{\partial G}{\partial \nu}\right)\right\|_{L^{2}\left(\Gamma_{e} \times \Gamma_{i}\right)}}{\|1\|_{L^{2}\left(\Gamma_{e} \times \Gamma_{i}\right)}},
$$

and

$$
\left(\Lambda_{\gamma}-\Lambda_{0}\right)\left(\Phi_{\omega}\right)+\frac{p}{100} \operatorname{rand}(1) \frac{\left\|\left(\Lambda_{\gamma}-\Lambda_{0}\right)\left(\Phi_{\omega}\right)\right\|_{L^{2}\left(\Gamma_{e} \times \Gamma_{i}\right)}}{\|1\|_{L^{2}\left(\Gamma_{e} \times \Gamma_{i}\right)}},
$$

where $\operatorname{rand}(1)$ is the random number in $(-1,1)$. When we consider the modal parameter measurement, we add $p \%$ noise to the eigenvectors, $v_{0}$ and $v_{\epsilon}$.

Example 1. In this example, we compare the numerical results of three different methods when there is a single corrosive part $I_{1}$. We take $\omega=5$ in the case of ultrasound measurements. Figure 2 summarizes the actual and computational results with noise $=$ $0 \%, 1 \%, 5 \%, 10 \%$, respectively. The results show that the MUSIC algorithms using electrostatic and ultrasound measurements detect the location pretty well. Even if the performance of the vibration test is still satisfactory, it is worst among three method. This is natural in a sense that the vibration test uses a single measurement of modal parameters while the other two use multiple measurements.

Example 2. In this example, we consider the case where there are three corrosive parts. Since the vibration test is difficult to apply to the case with multiple corrosive parts, we use only the electrostatic and ultrasound measurements, and compare the computational results. The actual data is summarized in the top of Figure 3. The table shows the computational results under noise $=0 \%, 1 \%, 5 \%, 10 \%$. It is interesting to find that those corrosive parts which are close to each other are detected as a single one as the noise level increases.

H.A. is partially supported by the Brain Pool Korea Program at Seoul National University, H.K. is partially supported by the grant KOSEF R01-2006-000-10002-0, and E.K. is supported by BK21 Math. division at Seoul National University. 


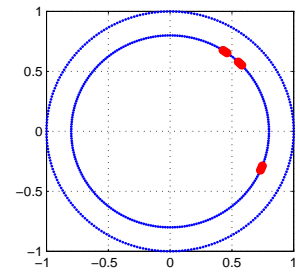

\begin{tabular}{|cccccc|}
\multicolumn{7}{|c}{ Actual data } \\
\hline $\mathrm{m}$ & $\omega$ & $\gamma_{k}$ & $z_{k}$ & $\langle\gamma\rangle_{k}$ & $\left|I_{k}\right|$ \\
\hline 3 & 1 & 0.1 & $(0.5372,0.5928)$ & 0.0059 & 0.0589 \\
& & 0.05 & $(0.4445,0.6652)$ & 0.0029 & 0.0589 \\
& & 1 & $(0.7391,-0.3061)$ & 0.0589 & 0.0589 \\
\hline
\end{tabular}

Detected data

\begin{tabular}{|c|ccc|ccc|}
\hline & \multicolumn{3}{|c|}{ Electrostatic M. } & \multicolumn{3}{c|}{ Ultrasound M. } \\
\hline noise(\%) & $m^{c}$ & $\left|z_{k}-z_{k}^{c}\right|$ & $\left|\langle\gamma\rangle_{k}-\langle\gamma\rangle_{k}^{c}\right|$ & $m^{c}$ & $\left|z_{k}-z_{k}^{c}\right|$ & $\left|\langle\gamma\rangle_{k}-\langle\gamma\rangle_{k}^{c}\right|$ \\
\hline 0 & 3 & 0.0000 & $4.4419 \mathrm{e}-5$ & 2 & 0.0393 & $2.8423 \mathrm{e}-3$ \\
& & 0.0000 & $1.5578 \mathrm{e}-5$ & & & \\
& & 0.0000 & $4.8254 \mathrm{e}-4$ & & 0.0000 & $3.0922 \mathrm{e}-3$ \\
\hline 1 & 3 & 0.0000 & $3.4664 \mathrm{e}-5$ & 2 & 0.0196 & $2.8608 \mathrm{e}-3$ \\
& & 0.0000 & $2.7453 \mathrm{e}-6$ & & & \\
& & 0.0000 & $4.7460 \mathrm{e}-4$ & & 0.0000 & $3.1920 \mathrm{e}-3$ \\
\hline 5 & 2 & 0.0000 & $2.3172 \mathrm{e}-3$ & 2 & 0.0393 & $2.8293 \mathrm{e}-3$ \\
& & 0.0000 & $4.4258 \mathrm{e}-4$ & & 0.0000 & $3.0990 \mathrm{e}-3$ \\
\hline 10 & 2 & 0.0196 & $2.2691 \mathrm{e}-3$ & 2 & 0.0393 & $2.8163 \mathrm{e}-3$ \\
& & 0.0000 & $4.0228 \mathrm{e}-4$ & & 0.0000 & $3.1058 \mathrm{e}-3$ \\
\hline
\end{tabular}

FiguRE 3. The computational results in the case of multiple corrosive parts.

\section{REFERENCES}

[1] H. Ammari, H. Kang, E. Kim, H. Lee, and K. Louati, Vibration testing for detecting internal corrosion, Stud. Appl. Math. 122(1) (2009), 85-104.

[2] H. Ammari, H. Kang, E. Kim, M. Lim, and K. Louati, Ultrasound imaging of internal defects, in preparation.

[3] H. Ammari, H. Kang, E. Kim, K. Louati, and M. Vogelius, A MUSIC-type algorithm for detecting internal corrosion from electrostatic boundary measurements, Numer. Math., 108 (2008), 501-528.

[4] H.T. Banks, M.L. Joyner, B. Wincheski, and W.P. Winfree, Real time computational algorithms for eddy-current-based damage detection, Inverse Problems, 18 (2002), 795-823.

[5] G. Buttazzo and R.V. Kohn, Reinforcement by a thin layer with oscillating thickness, Appl. Math. Opt., 16 (1988), 247-261.

[6] D. Colton and R. Kress, Inverse Acoustic and Electromagnetic Scattering Theory, 2nd ed. Berlin, Germany: Springer-Verlag, 1998.

[7] G. Inglese, An inverse problem in corrosion detection, Inverse Problems, 13 (1997), 977-994.

[8] P. Kaup and F. Santosa, Nondestructive evaluation of corrosion damage using electrostatic measurements, J. Nondestructive Eval. 14 (1995), 127-136.

[9] P. Kaup, F. Santosa, and M. Vogelius, A method for imaging corrosion damage in thin plates from electrostatic data, Inverse Problems, 12 (1996), 279-293.

[10] B. Luong and F. Santosa, Quantitative imaging of corrosion inplates by eddy current methods, SIAM J. Appl. Math., 58 (1998), 1509-1531.

[11] M. Vogelius and J. Xu, A nonlinear elliptic boundary value problem related to corrosion modelling, Quart. Appl. Math., 56 (1998), 479-505. 